\title{
Orientação para o Mercado Interno no serviço público de ensino
}

\author{
Internal Market Orientation \\ in the education public sector
}

\author{
ZÂMORA CRISTINA DOS SANTOS* \\ ROMULO MATOS DE MORAES** \\ EMERSON WAGNER MAINARDES *** \\ JOSÉ MARCOS NUNES BENEVENUTE****
}

\section{RESUMO}

O presente estudo é baseado na teoria do Marketing Interno (MI) e sua aplicação por meio da Orientação para o Mercado Interno (OMI) no contexto do serviço público. O objetivo é identificar quais fatores da OMI afetam a satisfação dos servidores de uma instituição de ensino pública de forma a contribuir com as políticas públicas. Trata-se de uma pesquisa descritiva, com corte transversal e abordagem quantitativa. A investigação empírica ocorreu a partir de um questionário estruturado com base no modelo de Gounaris (2006), respondido por 405 servidores da instituição pública de ensino. Os resultados obtidos revelaram que o serviço público tem baixa orientação para o mercado interno, mostrando que satisfação no trabalho e OMI são conceitos inter-relacionados, mas que, para o serviço público, existem outras variáveis que impactam a satisfação, porém não estão contempladas no modelo. Dos dez fatores testados, apenas os

* Instituto Federal do Espirito Santo. Mestre em Administração pela Fucape Business School. Especialista em Psicologia Organizacional e Recursos Humanos. Coordenadora de Extensão Ifes- Campus Aracruz. zamora@ifes.edu.br

** Instituto Federal do Espirito Santo. Mestre em Administração de Empresas pela Fucape Business School. Diretor do Departamento de Administração Ifes - Campus de Alegre. romulomoraes@hotmail.com .

*** Fucape Business School. Doutor em Administração pela Universidade da Beira IInterior -Portugal. Especialista em Administração e Marketing. Professor Associado e Pesquisador. emerson@fucape.br.

**** Instituto Federal do Espirito Santo. Mestre em Administração de Empresas pela Fucape Business School. jmbenevenute@ifes.edu.br 
seguintes mostraram-se significativos para satisfação do servidor: comunicação interna com os colaboradores; comunicação interna entre gerentes; e atenção aos grupos com necessidades comuns. Palavras-chave: Marketing Interno; Orientação para o Mercado Interno; setor público; satisfação no trabalho.

\section{Abstract}

The present study is based on the Internal Marketing (IM) theory and its application through the Internal Market Orientation (IMO) in the context of the public service. The aim is to identify which IMO factors affect the public employee's satisfaction of an educational institution in order to contribute to public policies. This is a descriptive study, with a cross-sectional and quantitative approach. The empirical research was carried out through a structured questionnaire based on the model of Gounaris (2006), answered by 405 servers of the studied institution. The results indicated that the public service has low exposure for internal market, showing that job satisfaction and IMO are interrelated concepts, but for the public context there are other variables that affect satisfaction, yet are not addressed in the model. Only the following factors were significant: internal communication with employees; internal communication between managers; and attention to groups with common needs.

Keywords: Internal Marketing; Internal Market Orientation; public sector; job satisfaction.

\section{INTRODUÇÃo}

A partir de 1980, a necessidade de uma reforma do Estado afetou vários países, um advento oriundo das crises financeiras e do baixo desempenho do serviço público (POLLITT; BOUCKAERT, 2011). No Brasil, essa reforma gerencial iniciou-se a partir de 1995, o que reestruturou as funções do Estado, tendo destaque os recursos humanos, com a exigência de qualificação e quantidade adequada de servidores movidos pelo compromisso com resultados, ou seja, satisfeitos com o seu trabalho (PACHECO, 2014).

Os servidores compõem o mercado interno das organizações públicas. A satisfação desse "cliente interno", como é chamado por Gummesson (1987), é debatida por meio da visão apresentada pelo 
Marketing Interno (MI), que concentra no capital humano a principal fonte da criação de valor para o público externo; a satisfação do cliente externo dependerá de satisfazer às necessidades desse público interno (GREMLER; JO BITNER; EVANS, 1994; GOUNARIS, 2010; ABZARI; GHORBANI; MADANI, 2011).

A Orientação para o Mercado Interno (OMI) é utilizada como um recurso para o desenvolvimento do MI, pois a valorização do funcionário tem sido um dos caminhos encontrados para melhorar o serviço público (GOUNARIS, 2006; ANDREWS; BOYNE, 2010), para superar resistência organizacional à mudança (ABZARI; GHORBANI; MADANI, 2011) e para elevar os níveis de satisfação dos colaboradores (LINGS; GREENLEY, 2005).

A maioria das pesquisas empíricas de OMI são em setores privados, como as realizadas por Rafiq e Ahmed (1993), Gounaris (2006), Grayson e Sanchez-Hernandez (2010), Acar et al. (2012) e Altarifi (2014). Poucas se dedicaram ao setor público (EWING; CARUANA, 1999; ZAMPETAKIS; MOUSTAKIS, 2007; MAINARDES; CERQUEIRA, 2015), apesar da abrangência e diversidade dos serviços e órgãos desse setor como saúde, segurança, moradia e aposentadoria. Ainda assim, compreender a satisfação do servidor público continua objeto de pesquisa predominante em estudos recentes (FOGAÇA; COELHO JUNIOR, 2015). Nesse sentido, é relevante analisar a OMI no contexto público brasileiro, tendo como amostra a área de educação.

Após as discussões e lacunas aqui apresentadas, o objetivo deste estudo foi identificar os fatores da Orientação para o Mercado Interno (OMI) que afetam a satisfação com o trabalho dos servidores de uma instituição pública de ensino.

Academicamente, a OMI vem sendo tema recente de publicações, porém constata-se que existe a oportunidade de ampliar as pesquisas e aplicações práticas, visto existirem poucos estudos com esse propósito como expõem, por exemplo, Ahmed e Rafiq (2003), Gounaris (2006), De Farias (2010) e Amangala e Amangala (2013). Ao considerar que o setor público de cada país tem a própria cultura e limitações, prestando diversos tipos de serviços em áreas distintas (POLLITT; BOUCKAERT, 2011), este trabalho pretende contribuir para a ampliação do conhecimento acerca dos fatores que afetam a satisfação de servidores de instituições públicas de ensino. 
Quanto à relevância social do estudo, espera-se que os resultados colaborem na formulação de políticas que considerem a OMI, uma vez que a satisfação no trabalho nas instituições de ensino possui efeito significativo no aumento na satisfação do aluno (ALTARIFI, 2014) e na qualidade dos serviços públicos prestados à sociedade (BERRY; HENSEL; BURKE, 1976).

\section{FUNDAMENTAÇÃo TEÓRICA}

\subsection{Marketing interno e serviço público}

A principal função da iniciativa pública é prestar serviço de qualidade à sociedade, e isso só é possível mediante a contribuição dos funcionários (ACAR et al., 2012), que precisam estar satisfeitos, visto que são eles que cumprirão esse papel (GREMLER; JO BITNER; EVANS, 1994).

O primeiro conceito em relação ao Marketing Interno envolve considerar os funcionários como primeiro mercado a ser atendido pela organização (SASSER; ARBEIT, 1976). Tem-se o entendimento de que as transações internas entre as organizações e seus empregados são tão importantes quanto as interações externas com o cliente, o que amplia as possibilidades de melhoria nos níveis de serviço (BERRY, 1987). Sob esse aspecto, os servidores e setores de uma organização pública passam a ser vistos como fornecedores e consumidores, trabalhando em sintonia para defender e apoiar a estratégia e os objetivos da organização pública (DE FARIAS, 2010).

A principal função do Marketing Interno é focada na motivação dos funcionários para o alcance dos resultados da organização (BERRY, 1987; EWING; CARUANA, 1999). Rafiq e Ahmed (1993) reforçam o conceito apontando ainda que o Marketing Interno se preocupa em utilizar técnicas para motivar os empregados, facilitando assim a implementação de estratégias e programas. No ambiente educacional não é diferente, em sua pesquisa, Altarifi (2014) comprovou que o MI é significativo em relação à satisfação no trabalho em Instituições de Ensino Superior, pois auxilia no nível do comprometimento organizacional (ACAR et al., 2012).

Observa-se uma ligação direta entre MI e a área de recursos humanos (RH), haja vista que a concepção principal do Marketing Interno é considerar os recursos humanos como o ativo mais valioso 
da organização (COLLINS; PAYNE, 1991; EWING; CARUANA, 1999; ABZARI; GHORDANI; MADANI, 2011). No caso do setor público, Ewing e Caruana (1999), ao analisarem a relação entre RH e MI em uma instituição pública australiana, reforçam a necessidade de orientação para o mercado em toda a instituição de forma a criar uma interface com a política de Gestão de Recursos Humanos com vistas a viabilizar as estratégias globais dela. Constata-se novamente uma cadeia na qual a Orientação para o Mercado Interno contribui para a eficácia da Gestão de Recursos Humanos e este sustenta as ações voltadas á sociedade

Com foco no atendimento à sociedade, o setor público vem reestruturando seus procedimentos burocráticos em busca de melhores indicadores de desempenho e produtividade (POLLITT; BOUCKAERT, 2011), com modelos de gestão orientados para o mercado externo (WALKER et al., 2011). Nesse contexto, o Marketing Interno vem com a função extra de assegurar satisfação do empregado (GOUNARIS, 2008), visando à satisfação do cliente externo ou cidadão (GRONROOS, 1981; BERRY, 1987). Práticas como essas podem ainda produzir outros efeitos como o empreendedorismo público e, por consequência, a busca por inovação (ZAMPETAKIS; MOUSTAKIS, 2007).

\subsection{Orientação para o Mercado Interno}

Para efetividade das ações do Marketing Interno é necessário desenvolver iniciativas focadas que possuam o alcance suficiente para convergir internamente à empresa e seus propósitos. É fundamental que tais propostas sejam precedidas de uma orientação interna que possibilite sua realização (GOUNARIS, 2006).

Com a Orientação para o Mercado Interno (OMI), a organização busca entender e responder às necessidades de seus clientes internos, de forma que, após avaliar a percepção da relação de troca existente entre funcionário/empregador, seja possível propor ações de marketing capazes de melhorar os níveis de satisfação e comprometimento (LINGS; GREENLEY, 2005; GOUNARIS, 2008). A utilização da OMI oportuniza à empresa organizar suas práticas de MI e programar ações efetivas que atendam aos servidores (GOUNARIS, 2008).

O trabalho de Gounaris, Vassilikopoulou e Chatzipanagiotou (2010) sobre OMI mostra que o valor do cliente continua primordial 
para a empresa, porém deixa evidente que o compromisso dela foi ampliado para gerar valor às diferentes partes interessadas (stakeholders). Aprofundando-se no entendimento de Gounaris, Vassilikopoulou e Chatzipanagiotou (2010), percebe-se que marketing orientado externamente e internamente estão interligados, ao propor ações capazes de gerar maior valor aos empregados, o que refletirá na qualidade de serviço e nos níveis de qualidade percebidos pelos clientes.

Foi identificada uma relação positiva da OMI com participação na tomada de decisões, empoderamento e satisfação no trabalho, sendo este último um fator-chave para apurar o desempenho da organização quanto à OMI, já que as metas de programas de Marketing Interno têm nesse componente alto grau de importância (GOUNARIS, 2006).

Para mensurar a Orientação para o Mercado Interno, Gounaris (2006) identificou, testou e validou um instrumento composto por três grandes dimensões seguidas de dez subdimensões ou fatores, termo que será o utilizado neste estudo. A base foi o estudo de Kohli e Jaworski (1990), que apontou três pilares do MI: a produção de conhecimento sobre os servidores, a disseminação desse conhecimento e sua resposta ao conhecimento sobre os servidores. Com base neles, Lings (2004) procurou descrever em sua pesquisa os componentes correspondentes, facilitando a mensuração da OMI e oferecendo base à abordagem hierarquizada proposta por Gounaris (2006). O Quadro 1 apresenta essas dimensões e subdimensões juntamente com a codificação a ser utilizada na análise dos dados.

Quadro 1: Orientação para o Mercado Interno

\begin{tabular}{|l|l|}
\hline Dimensões & Subdimensões (Fatores) \\
\hline Geração e conhecimento & GC1 - Identificação das necessidades do cliente interno \\
dos empregados (GC) & GC2 - Condições de mercado de trabalho \\
& GC3 - Atenção a grupos com necessidades comuns \\
& GC4 - Atenção aos servidores \\
\hline $\begin{array}{l}\text { Disseminação do } \\
\text { conhecimento (DC) }\end{array}$ & DC1 - Comunicação interna com os colaboradores \\
\hline RC2 - Comunicação entre gerentes \\
conhecimento ao \\
gerado (RC)
\end{tabular}$\quad \begin{aligned} & \text { RC1 - Descrição dos cargos } \\
& \text { RC2 - Sistema de remuneração } \\
& \text { RC3 - Treinamento } \\
& \text { RC4 - Conhecimento gerencial }\end{aligned}$

Fonte: Gounaris (2006). Adaptado pelos autores. 
Para mensurar o grau de OMI, é necessário entender como o conhecimento dos gestores sobre seus servidores é conduzido. As análises extraídas das subdimensões podem ser um instrumento valioso na identificação das necessidades e dos motivos de insatisfação do mercado interno, permitindo aos gestores elaborarem políticas que atendam às necessidades e expectativas de seus funcionários. Dessa forma, há possibilidade de ampliar o entendimento sobre seu corpo funcional e convergir em objetivos, tendo, como resultante, ganho de desempenho e criação de vantagem competitiva para a instituição pública (CONDUIT; MAVONDO, 2001; GOUNARIS, 2008).

\section{Metodologia}

Dado o objetivo do estudo, o método utilizado nesta pesquisa foi o quantitativo, descritivo, com corte transversal, no intuito de identificar os fatores de OMI que afetam a satisfação com o trabalho dos servidores de uma instituição pública de ensino.

A população da pesquisa compôs-se de servidores do setor da educação pública, pois a literatura existente apresenta poucas pesquisas empíricas nesse segmento (BOYNE, 2004). A escolha do Instituto Federal de Educação do Espírito Santo (IFES) como campo de estudo se deve ao fato de fazer parte de uma rede federal de institutos presente em todo o território nacional e de ser a instituição de ensino pública com maior número de servidores na região.

A amostra, caracterizada por ser não probabilística por conveniência (MATTAR, 1996), foi representada por docentes e técnicos administrativos da instituição de ensino. $\mathrm{O}$ instrumento de coleta dos dados foi encaminhado, por e-mail, para um total de 2.448 servidores ativos no ano de 2015. A amostra se deu pelos 405 respondentes, entre técnicos administrativos e docentes. O questionário estruturado seguiu o modelo aplicado por Gounaris (2006), com dez subdimensões (ou fatores) composto de 43 afirmações. Para o presente estudo, dado que a população se refere ao mercado interno do setor público, algumas afirmações não se aplicavam e foram retiradas:

- 1 afirmação da subdimensão Identificação das expectativas dos servidores, que tratava dos concorrentes, visto que o Instituto não tem concorrente direto. 
- 3 afirmações da subdimensão Condições do mercado de trabalho, que abordavam sistema jurídico, que é especifico de cada carreira no serviço público, Taxa de desemprego na área, que não se aplica ao serviço público, além da Concorrência.

- 4 afirmações da subdimensão Remuneração, visto não haver bonificação ou remuneração proporcional ao desempenho no serviço público.

O questionário final foi composto por 36 afirmações acompanhadas de uma escala de Likert com cinco pontos (MALHOTRA, 2012) e construído em duas partes. A primeira foi composta por 1 afirmação relacionada à satisfação no trabalho (variável dependente) e 35 afirmações agrupadas dentro das dimensões e subdimensões. A segunda parte se compôs das variáveis de controle, ou seja, o perfil dos respondentes, onde foram desenvolvidas questões referentes a gênero, idade, carreira, tempo de serviço, formação e função de chefia.

No processo de análise dos dados, primeiramente foi realizada a caracterização da amostra medida pela proporção de categorias sociodemográficas. Na sequência foi feita a estatística descritiva básica de cada variável, as afirmações de sentindo oposto foram invertidas para que a média não fosse comprometida. Em um segundo momento, foi utilizada regressão linear múltipla, cuja variável dependente é a satisfação (Y) e a independente as dez subdimensões/ fatores (CG1; CG2; CG3; CG4; DC1; DC2; RC1; RC2; RC3; RC4), conforme ilustrado anteriormente no Quadro 1.

\section{ANÁLISE DE DADOS}

\subsection{Caracterização da amostra}

A Tabela 1 refere-se aos dados da caracterização da amostra, obtida por meio das questões de perfil demográfico. 
Tabela 1: Dados demográficos da amostra

\begin{tabular}{llll}
\hline \multirow{2}{*}{ CATEGORIAS } & DIVISÕES & $\begin{array}{l}\text { QUANTI- } \\
\text { DADE }\end{array}$ & $\begin{array}{l}\text { PER- } \\
\text { CEN- } \\
\text { TUAL }\end{array}$ \\
\hline \multirow{2}{*}{ 1. Gênero } & Masculino & 219 & $54,10 \%$ \\
& Feminino & 186 & $45,90 \%$ \\
\hline \multirow{2}{*}{ 2. Carreira } & Docente & 126 & $31,10 \%$ \\
& Técnico administrativo & 279 & $68,90 \%$ \\
\hline \multirow{2}{*}{ Exerce cargo } & Sim & 90 & $22,20 \%$ \\
de chefia & Não & 315 & $77,80 \%$ \\
\hline \multirow{4}{*}{ 4.Tempo de } & Menos de 1 ano & 32 & $07,90 \%$ \\
Instituto & Acima de 1 ano até 3 anos & 84 & $20,70 \%$ \\
& Acima de 3 anos até 5 anos & 72 & $17,80 \%$ \\
& Acima de 7 anos até 7 anos & 71 & $17,50 \%$ \\
& Ensino Fundamental & 146 & $36,00 \%$ \\
\hline \multirow{2}{*}{ instrução } & Ensino Médio/Técnico & 05 & $01,20 \%$ \\
& Superior completo/incompleto & 49 & $12,20 \%$ \\
& Pós-graduação & & $13,30 \%$ \\
& completa/incompleta & 126 & $31,30 \%$ \\
& Mestrado completo/incompleto & 121 & $29,90 \%$ \\
& & & \\
& Doutorado completo/incompleto & 50 & $12,30 \%$ \\
\hline
\end{tabular}

Fonte: Dados da pesquisa.

Quanto ao gênero, na amostra analisada verificou-se predominância do masculino com $54,1 \%$ contra $45,9 \%$ feminino. Esse fato pode ser explicado devido a muitos campi possuírem características de áreas agrícolas, com grande extensão territorial e intensa atividade agropecuária, sendo desenvolvida por número acentuado de servidores do gênero masculino. O estudo realizado por Mainardes e Cerqueira (2015) junto ao corpo de bombeiros corrobora a predominância masculina em algumas áreas do serviço público por questões relacionadas à cultura do ambiente de trabalho e a limitações físicas do gênero feminino. 
Evidenciou-se que a maior parte dos respondentes faz parte do corpo técnico-administrativo, responsável por $68,9 \%$ dos questionários respondidos, contra $31,1 \%$ dos docentes. Acredita-se que o percentual elevado de respondentes técnico-administrativos se explica pelo fato de estarem maior tempo com acesso à internet devido às suas atividades administrativas.

Verificou-se que $77,8 \%$ dos respondentes não possuem cargo de chefia e $36 \%$ são servidores que atuam há mais de sete anos no Instituto, compondo a maioria dos respondentes. Portanto, a amostra foi constituída por servidores com experiência e bom conhecimento das políticas praticadas dentro do Instituto e que podem fornecer respostas mais precisas e próximas da realidade objetivada na pesquisa. Também ficou evidente que o grau de instrução dos servidores é elevado: 31,30\% composto por pessoas com pós-graduação completa/incompleto, 29,9\% com mestrado completo/incompleto e $12,30 \%$ com doutorado completo/incompleto; somente $1,20 \%$ possui apenas o nível fundamental, e 12,20\% com ensino médio ou técnico.

A amostra selecionada mostrou-se válida em todas as variáveis de controle, exceto pelo fato de os técnicos administrativos apresentarem um volume maior de respostas, apesar de o quadro de servidores do Instituto ser equilibrado entre as carreiras. As demais variáveis foram similares ao servidor médio do IFES: predominância masculina, escolaridade de pós-graduação, tempo de serviço menor de dez anos e em sua grande maioria não exerce cargo de chefia.

\subsection{Estatística descritiva}

Os dados referidos na Tabela 2 apresentam a média e os desvios-padrão dos dez fatores analisados, resultantes das assertivas do questionário. Na dimensão Geração de Conhecimento encontram-se quatro fatores, dos quais a Identificação das expectativas dos servidores (GC1) foi o que obteve desempenho superior aos demais, com média de 2,90 e um desvio-padrão de 0,92, seguido pela Situação do Mercado de Trabalho (GC2,) com média de 2,78 e desvio-padrão de 1,00. O menor escore entre os quatro fatores dessa dimensão foi obtido na Atenção aos Grupos com Necessidades Comuns (GC3) (média de 2,43 e 0,72 de desvio-padrão), seguido pela Atenção ao Público Interno (GC4) (média 2,49 e desvio-padrão de 0,73). 
Os resultados apurados na Dimensão Geração do Conhecimento revelaram que, na visão dos respondentes, o Instituto não procura compreender as necessidades dos servidores. Para os participantes da pesquisa, o IFES tende a identificar as necessidades genericamente, não priorizando aquelas de grupos específicos. De acordo com estudos de Gounaris (2006) e Lings (2004), esses resultados terão como consequência a elaboração de políticas de recursos humanos abrangentes, de forma a desconsiderar as peculiaridades dos diversos grupos existentes dentro da organização.

Tabela 2: Média e desvio-padrão das variáveis pesquisadas

\begin{tabular}{|c|c|c|c|}
\hline Dimensões & $\begin{array}{l}\text { Fatores/Subdimensões } \\
\text { (Variáveis independentes) }\end{array}$ & Média & Desvio-padrão \\
\hline \multirow{4}{*}{$\begin{array}{l}\text { Geração e conheci- } \\
\text { mento dos emprega- } \\
\text { dos (GC) }\end{array}$} & $\mathrm{GC} 1$ & 2,90 & 0,92 \\
\hline & GC2 & 2,78 & 1,00 \\
\hline & GC3 & 2,43 & 0,72 \\
\hline & GC4 & 2,49 & 0,73 \\
\hline \multirow{2}{*}{$\begin{array}{l}\text { Disseminação do } \\
\text { conhecimento (DC) }\end{array}$} & $\mathrm{DC} 1$ & 3,14 & 0,95 \\
\hline & DC2 & 2,85 & 0,62 \\
\hline \multirow{4}{*}{$\begin{array}{l}\text { Respostas ao co- } \\
\text { nhecimento gerado } \\
\text { (RC) }\end{array}$} & $\mathrm{RC} 1$ & 3,11 & 0,79 \\
\hline & $\mathrm{RC} 2$ & 2,65 & 1,23 \\
\hline & RC3 & 2,73 & 0,70 \\
\hline & RC4 & 2,64 & 1,01 \\
\hline $\begin{array}{l}\text { Variável dependen- } \\
\text { te: Satisfação }(Y)\end{array}$ & & 3,56 & 1,08 \\
\hline
\end{tabular}

Fonte: Dados da pesquisa.

Analisando a dimensão Disseminação de Conhecimento na Tabela 2, o fator que mostrou maior média foi a Comunicação entre as chefias e servidores (DC1), com 3,14 e desvio-padrão de 0,95, ao passo que o fator Comunicação entre Gerentes (DC2) teve média de 2,85 e desvio-padrão de 0,62. Isso demonstra que, na percepção dos servidores, a comunicação entre os níveis hierárquicos é regular, mas deficiente na comunicação horizontal. Segundo Lings (2004), a 
relação procedente da disseminação da informação para o público interno, de forma universalizada, reforça o apego do servidor com o Instituto.

Na terceira dimensão estudada, Resposta ao Conhecimento Gerado, o fator Descrição dos Cargos (RC1) foi a que apresentou a média mais elevada: 3,11 e desvio-padrão de 0,79 . Os servidores consideram de maneira regular as atividades que desempenham em relação a suas necessidades e objetivos. Isso indica que ainda cabem ajustes na descrição de trabalho, pois as organizações que optam por atividades mais ajustadas desenvolvem uma força de trabalho mais flexível, capaz de adaptar-se às novas necessidades (GRAYSON; SANCHEZ-HERNANDEZ, 2010).

Continuando a análise da dimensão citada anteriormente, o fator relacionado à remuneração (RC2), com uma única afirmativa, obteve a média de 2,65 e desvio-padrão de 1,23 . O resultado demonstra que grande parcela de servidores acredita que suas rendas não estão vinculadas às suas qualificações, e nesse caso é possível sugerir que o governo necessita estruturar a política de remuneração de forma mais universalizada e atrativa para esse público, uma vez que remuneração é parte do reconhecimento de valor que o servidor identifica na sua prestação de serviço ao Instituto (LINGS; GREENLEY, 2005).

O fator Treinamento (RC3) obteve média de 2,73 e desvio-padrão de 0,70. Esses dados mostram que, para a maioria dos servidores, os treinamentos não atendem às suas demandas, daí a necessidade de que a administração colete mais informação de seus funcionários para que consiga fornecer uma formação que os satisfaçam e os motive (AMANGALA; AMANGALA, 2013).

Na análise da última subdimensão ou fator Conhecimento Gerencial (RC4), na Tabela 2, ainda dentro da terceira dimensão, observaram-se as menores médias dessa dimensão, com 2,64 e desvio-padrão de 1,01. Isso reflete que para grande parcela ainda não existe uma preocupação da alta administração com os servidores, ou seja, as necessidades do "cliente interno" não estão sendo consideradas pelo Instituto (BERRY; HENSEL; BURK, 1976).

Quanto à variável dependente Satisfação no trabalho $(Y)$, foi a que obteve a maior média desse estudo $(3,56)$, com desvio-padrão 
de 1,08. Tal resultado deixou claro que, mesmo necessitando de melhorias nas políticas de recursos humanos, política salarial e maior percepção de valor dos servidores em relação à alta gestão, os servidores demonstram estarem satisfeitos em trabalhar no Instituto/serviço público.

As médias mostraram-se em sua maioria regulares ou baixas - não houve nenhuma superior a 4,0 entre os fatores. O serviço público analisado apresenta deficiências significativas em algumas áreas: entender as necessidades dos servidores, comunicação com os gestores, remuneração, treinamento e o interesse gerencial.

\subsection{Regressão linear múltipla}

O resumo do modelo, juntamente com os resultados dos testes aplicados, é apresentado na Tabela 4 . Pode-se perceber que o $\mathrm{R}^{2}$ ajustado indica que a variável dependente (satisfação com o trabalho) pode ser explicada por 19\% das covariáveis (os fatores testados), conforme pode ser visto na Tabela 5 . O modelo F possui significância de .003 , sendo $\mathrm{P}<0.05$, significativo a $95 \%$.

Tabela 4: Resumo do modelo

\begin{tabular}{|c|c|c|c|c|c|c|c|c|}
\hline \multirow[b]{2}{*}{$\mathrm{R}$} & \multirow[b]{2}{*}{$\mathrm{R}^{2}$} & \multirow{2}{*}{$\begin{array}{l}\mathrm{R}^{2} \text { ajus- } \\
\text { tado }\end{array}$} & \multirow{2}{*}{$\begin{array}{l}\text { Erro } \\
\text { padrão do } \\
\text { estimador }\end{array}$} & \multicolumn{4}{|c|}{ Estatísticas de Mudança } & \multirow{2}{*}{$\begin{array}{l}\text { Durbin- } \\
\text {-Watson }\end{array}$} \\
\hline & & & & $\begin{array}{l}\text { Mudança } \\
\text { de } \mathrm{R}^{2}\end{array}$ & $\mathrm{~F}$ & df1 df2 & $\begin{array}{l}\text { Sig. } \\
\text { F }\end{array}$ & \\
\hline, $443^{*}$ & 0,196 & 0,19 & 0,97421 & 0,017 & 8,714 & 401 & 0,003 & 1,789 \\
\hline
\end{tabular}

Teste de Aderência Kolmogorov-Smirnov: suporta hipótese de aderência à distribuição normal

Teste de Aleatoriedade: suporta a hipótese de aleatoriedade

Teste de Homocedasticidade: suporta a hipótese de homocedasticidade Anova: Significativo

* Preditores: (Constante), DC1, GC3, DC2

No processo de estimação, sete variáveis independentes não mostraram significância para o presente estudo. A Tabela 5 apresenta as subdimensões/fatores do modelo de Gounaris (2006) que foram significativas ao nível de 5\%. No modelo os valores apresentados 
para o fator de inflação da variância (VIF) são aceitáveis para rejeitar problemas de multicolinearidade entre as medidas independentes, considerando-se 5 o valor máximo tolerável (HAIR et al., 2005).

Tabela 5: Coeficientes

\begin{tabular}{|c|c|c|c|c|c|c|c|c|c|c|c|}
\hline \multirow{3}{*}{ Modelo } & \multirow{2}{*}{\multicolumn{2}{|c|}{$\begin{array}{l}\text { Coeficientes } \\
\text { Despadron. }\end{array}$}} & \multirow{3}{*}{\begin{tabular}{|l} 
Coefic. \\
Padron.
\end{tabular}} & \multirow{3}{*}{$\mathrm{T}$} & \multirow{3}{*}{ Sig. } & \multirow{2}{*}{\multicolumn{2}{|c|}{$\begin{array}{l}95,0 \% \text { intervalo } \\
\text { de confiança } \\
\text { para B }\end{array}$}} & \multirow{2}{*}{\multicolumn{3}{|c|}{ Correlações }} & \multirow{3}{*}{$\begin{array}{l}\text { Estatís- } \\
\text { tica } \\
\text { Coline- } \\
\text { arid. } \\
\text { VIF }\end{array}$} \\
\hline & & & & & & & & & & & \\
\hline & B & $\begin{array}{l}\text { Erro } \\
\text { padrão }\end{array}$ & & & & $\begin{array}{l}\text { Banda } \\
\text { baixa }\end{array}$ & $\begin{array}{l}\text { Banda } \\
\text { Alta }\end{array}$ & $\begin{array}{l}\text { Ordem } \\
\text { zero }\end{array}$ & Parcial & Part & \\
\hline (Const) & 1,328 & 0,253 & & 5,24 & 0 & 0,830 & 1,827 & & & & \\
\hline $\mathrm{DCl}$ & 0,300 & 0,060 & 0,264 & 5,00 & 0,000 & 0,182 & 0,417 & 0,392 & 0,242 & 0,22 & 1,39 \\
\hline GC3 & 0,234 & 0,076 & 0,159 & 3,09 & 0,002 & 0,085 & 0,383 & 0,326 & 0,152 & 0,14 & 1,32 \\
\hline DC2 & 0,249 & 0,084 & 0,144 & 2,95 & 0,003 & 0,083 & 0,414 & 0,287 & 0,146 & 0,13 & 1,18 \\
\hline
\end{tabular}

Legenda: DC1 - Comunicação interna com os colaboradores; GC3 - Atenção aos grupos com necessidades comuns; DC2 - Comunicação interna entre gerentes

Apenas os seguintes fatores mostraram-se associadas à satisfação com o trabalho no Instituto: Comunicação interna com os colaboradores (DC1); Comunicação interna entre gerentes (DC2); e Atenção aos grupos com necessidades comuns (GC3).

A pesquisa revelou que os três fatores (DC1, DC2 e GC3), associados à satisfação dos servidores, apresentaram médias baixas: DC1 $(\mathrm{M}=3,14)$, DC2 $(\mathrm{M}=2,85)$ e GC3 $(\mathrm{M}=2,49)$. Nenhum deles pode ser considerado como satisfatório, ou seja, na opinião dos servidores essas ações precisam ser melhoradas. Porém, apesar disso, os funcionários tendem a estar "Satisfeitos" com o trabalho (média 3,56).

Analisando os fatores DC1 e DC2, que compõem a dimensão Disseminação do Conhecimento sobre os empregados, tem-se a indicação de que há necessidade de melhora da comunicação interna, visto que tais variáveis estão associadas à satisfação. Segundo Grayson e Sanchez-Hernandez (2010), para que a disseminação de informação interna ocorra, são necessárias mensagens claras aos servidores, com ausência de ambiguidade; trata-se de uma variável importante, devido à capacidade em criar os vínculos entre servidores e gestores e melhorar a compreensão dos objetivos organizacionais pelos servidores (KELLER et al., 2006; ALTARIFI, 2014). 
Considerando que a comunicação é um fator-chave para o alcance da satisfação do servidor, é necessária a aplicação de um programa de marketing interno que sincronize a comunicação entre os níveis de gestão do Instituto (SOLIMAN, 2013). A criação de uma linha aberta entre chefe e chefiado permitirá que se conheçam os problemas e se compartilhem informações organizacionais. A troca de informação entre os gerentes possibilita compreender de forma coletiva os servidores e a proposição de solução por gestores de outros departamentos (GOUNARIS, 2006).

Quanto à dimensão Geração de conhecimento dos empregados, apenas a variável Atenção aos grupos com necessidades comuns (GC3) mostrou-se significativa, porém apresentou a menor média de toda a pesquisa. Isso é um indicativo de que, segundo os respondentes, o Instituto não observa os servidores como grupos com diferentes necessidades a serem satisfeitas. Por esse motivo, possivelmente não responderão da mesma forma aos incentivos, daí a necessidade de programas flexíveis e específicos por grupos, se o objetivo for o desenvolvimento do Marketing Interno pela organização (CAHILL, 1996). Nesse sentido, é essencial o reconhecimento por parte do Instituto da existência de duas carreiras diferentes (docentes e técnicos administrativos), que possuem características e demandas distintas (GOUNARIS, 2008) e que para satisfazê-las devem ser consideradas separadamente (AHMED; RAFIQ, 2003).

Ao comparar com os resultados obtidos por Mainardes e Cerqueira (2015), realizado também no serviço público, notou-se que cinco fatores foram significativos no estudo citado, contra três nesta pesquisa. A única variável significativa comum entre os dois estudos foi Atenção aos grupos com necessidades comuns, que obteve uma média baixa em ambos. Essa variável comum entre os estudos pode indicar que os servidores de forma geral valorizam terem as necessidades individuais identificadas, no entanto o setor público tende a homogeneizar as características dos servidores, tratando-os da mesma forma.

Em suma, o estudo mostrou que os fatores de Gounaris (2006) não explicaram de forma suficiente a satisfação do servidor público, visto que não representaram nem $1 / 3$ de implicação na variável dependente. Também foi possível identificar que as ações de OMI 
que se mostram relevantes à satisfação dos servidores da instituição analisada carecem de atenção por parte do Instituto, pois seu objetivo é elevar a satisfação do público interno e, em contrapartida, melhorar o serviço prestado à sociedade.

\section{CONCLUSÕES E RECOMENDAÇõeS}

O estudo objetivou identificar os fatores de OMI que afetam a satisfação do trabalho dos servidores de uma instituição pública de ensino. Constatou-se que apenas três dos dez fatores baseados em Gounaris (2006) tiveram significância na satisfação dos servidores: Comunicação interna com os colaboradores; Comunicação interna entre gerentes; e Atenção aos grupos com necessidades comuns. Isso mostra que se a instituição melhorar os processos de comunicação interna (tanto com colaboradores quanto entre gestores) e perceber as diferentes necessidades entre os servidores, a satisfação com o trabalho dos servidores tende a aumentar.

Os resultados mostram ainda a baixa Orientação para o Mercado Interno da instituição de ensino, visto que as médias se mantiveram entre 2 e 3 da escala de Likert (Discordo e Indiferente), corroborando o exposto por Mainardes e Cerqueira (2015) de que, na visão dos servidores, as organizações públicas demonstram pouco interesse no seu mercado interno.

A utilização do Marketing Interno baseado na Orientação para o Mercado Interno nas instituições públicas - e especificamente no contexto desta pesquisa - tem um papel fundamental no desenvolvimento do servidor e na forma como o serviço de educação é oferecido à sociedade. Afinal, se seu efeito for percebido, afetará diretamente a satisfação do trabalhador e, consequentemente, a prestação do serviço (GOUNARIS, 2006). Por outro lado, são necessárias mais investigações que explorem novas variáveis para ampliar a compreensão da OMI em organizações públicas.

Uma das principais limitações do estudo foi que o modelo explica apenas 19\% da satisfação dos servidores, mostrando que existem outras variáveis que afetam a satisfação e que não foram contempladas na pesquisa, daí a demanda por um aprofundamento nas pesquisas teórica e empírica no contexto do serviço público (EWING; CARUANA, 1999). Além disso, o trabalho aqui apresentado limitou- 
-se a pesquisar uma única organização de um setor entre os diversos tipos de organizações no setor público, e os resultados encontrados aqui podem não representar outras organizações públicas.

O estudo contribuiu teoricamente ao ampliar a literatura que investiga a OMI nas instituições - no caso aqui estudado, em uma organização pública de educação. Entender a OMI em todo tipo de organização poderá desenvolver o conceito, tornando-o uma abordagem teórica robusta (AHMED; RAFIQ 2003; GUMMESSON, 1987). Como contribuição prática à execução da pesquisa de OMI em uma instituição pública de ensino, o estudo reforçou que a OMI está associada à satisfação no trabalho nesse tipo de organização (ALTARIFI, 2014), permitindo assim sua aplicabilidade em outras instituições com esse perfil.

Para futuros estudos recomenda-se a continuidade na investigação da OMI em outra área do serviço público, com intuito da validação dos fatores. Também se sugere realizar uma análise da percepção das ações de OMI pelas diferentes carreiras em um mesmo órgão público. Propõe-se ainda averiguar a existência de outras possíveis variáveis relacionadas à OMI que afetam especificamente a satisfação do servidor público.

\section{REFERÊNCIAS}

ABZARI, M.; GHORBANI, H.; MADANI, F. A. The effect of internal marketing on organizational commitment from market-orientation viewpoint in hotel industry in Iran. International Journal of Marketing Studies, v. 3, n. 1, p. 147-155, feb. 2011. Disponível em: <http://www. ccsenet.org/journal/index.php/ijms/article/viewFile/9282/6848>. Acesso em: 12 de out de 2014.

ACAR, N.; SEKER, M.; BAYRAM, A.; ACAR, T. The Effect of Internal Marketing on the Performance of Employee and the Mediating Role of Organizational Commitment. ATINER'S Conference Paper Series, No: BUS2012-0215. Athens: Athens Institute for Education and Research, 2012. Disponível em: <http://www.atiner.gr/papers/BUS2012-0215.pdf>. Acesso em: 17 de out de 2014.

AHMED, P. K.; RAFIQ, M. Internal marketing issues and challenges. European Journal of marketing, v. 37, n. 9, p. 1177-1186, 2003. Disponível em: <http://dx.doi. org/10.1108/03090560310498813>. Acesso em: 12 de out de 2014.

ALTARIFI, S. Internal Marketing Activities in Higher Education. International Journal of Business and Management, v. 9, n. 6, p. 126-138, 2014. Disponível em: <http://www.ccsenet. org/journal/index.php/ijbm/article/view/35274/21204>. Acesso em: 06 de jan de 2015. 
AMANGALA, T.; AMANGALA, E. Effects of Internal Marketing and Its Components on Organisational Level Customer Orientation: An Empirical Analysis. European Journal of Business and Management, v. 5, n. 8, p. 64-73, 2013. Disponível em: <http://iiste.org/Journals/ index.php/EJBM/article/view/5097/5186>. Acesso em: 17 de out de 2014.

ANDREWS, R.; BOYNE, G. Better Public Services: The moral purpose of public management research? Discussants: Christopher Hood, Owen Hughes and Don Kettl. Public Management Review, v. 12, n. 3, p. 307-321, 2010. Disponível em: <http://www.tandfonline.com/doi/ pdf/10.1080/14719030903286656>. Acesso em: 25 de nov de 2014.

BERRY, L.; HENSEL, J. S.; BURKE, M. C. Improving retailer capability for effective consumerism response. Journal of Retailing, v. 52, n. 3, p. 3-14, 1976. Disponível em: <http://connection.ebscohost.com/c/articles/4681884/improving-retailer-capability-effective-consumerismresponse>. Acesso em: 10 de jan de 2015.

BERRY, L. Big Ideas in Services Marketing. Journal of Services Marketing, v. 1, n. 1, p. 5-9, 1987. Disponível em: <http://dx.doi.org/10.1108/eb008162>. Acesso em: 12 de jan de 2015.

BOYNE, G. A. Explaining public service performance: Does management matter? Public Policy and Administration, v. 19, n. 4, p. 100-117, 2004. Disponível em: <http://ppa.sagepub. com/content/19/4/100.abstract>. Acesso em: 14 de nov de 2014.

CAHILL, D. J. Internal marketing: Your company's next stage of growth. Routledge, 1996.

COLLINS, B.; PAYNE, A. Internal marketing: a new perspective for HRM. European Management Journal, v. 9, n. 3, p. 261-270, 1991. Disponível em: <http://www.sciencedirect.com/ science/article/pii/026323739190006C>. Acesso em: 17 de out de 2014.

CONDUIT, J.; MAVONDO, F. T. How critical is internal customer orientation to market orientation? Journal of Business Research, v. 51, n. 1, p. 11-24, 2001. Disponível em: <http:// dx.doi.org/10.1016/S0148-2963(99)00044-2>. Acesso em: 14 de nov de 2014.

DE FARIAS, S. A. Internal Marketing (IM): a literature review and research propositions for service excellence. Brazilian Business Review, v. 7, n. 2, p. 99-115, 2010. Disponível em: $<$ http://www.redalyc.org/articulo.oa?id=123021648006>. Acesso em: 10 de jan de 2015.

EWING, M. T.; CARUANA, A. An internal marketing approach to public sector management: the marketing and human resources interface. International Journal of Public Sector Management, v. 12, n. 1, p. 17-29, 1999. Disponível em: <http://dx.doi.org/10.1108/09513559910262652>. Acesso em: 17 de out de 2014.

FOGAÇA, N.; COELHO JUNIOR, F. A. A hipótese "trabalhador feliz, produtivo": o que pensam os servidores públicos federais / The assumption "happy, productive worker": what the federal civil servants think. Cadernos EBAPE. BR, v. 13, n. 4, p. 759, 2015.

GOUNARIS, S. P. Internal-market orientation and its measurement. Journal of Business Research, v. 59, n. 4, p. 432-448, 2006. Disponível em: <http://dx.doi.org/10.1016/j.jbusres.2005.10.003>. Acesso em: 10 de jan de 2015.

. The notion of internal market orientation and employee job satisfaction: some preliminary evidence. Journal of Services Marketing, v. 22, n. 1, p. 68-90, 2008. Disponível em: <http://dx.doi.org/10.1108/08876040810851978>. Acesso em: 12 de out de 2014. 
Internal-market orientation: a misconceived aspect of marketing theory. European Journal of Marketing, vol. 44, pp. 44-60, 2010. Disponível em: http://dx.doi. org/10.1108/03090561011079837>. Acesso em: 02 de nov de 2014.

GOUNARIS, S. P.; VASSILIKOPOULOU, A.; CHATZIPANAGIOTOU, K. C. Internal-market orientation: a misconceived aspect of marketing theory. European Journal of Marketing, v. 44, n. 11/12, p. 1667-1699, 2010. Disponível em: <http://dx.doi.org/10.1108/0309056101107983 7>. Acesso em: 14 de nov de 2014.

GRAYSON, D.; SANCHEZ-HERNANDEZ, M. I. Using internal marketing to engage employees in corporate responsibility. Journal of Cranfield University, v. 4, p. 401-414, 2010. Disponível em: <https://www.som.cranfield.ac.uk/som/dinamic-content/media/GRAYSON\%20and\%20 SANCHEZ\%20IM\%20FINAL\%20pdf.pdf $>$. Acesso em: 25 de nov de 2014.

GREMLER, D. D.; JO BITNER, M.; EVANS, K. R. The internal service encounter. International Journal of Service Industry Management, v. 5, n. 2, p. 34-56, 1994. Disponível em: <http:// dx.doi.org/10.1108/09564239410057672>. Acesso em: 06 de jan de 2015.

GRONROOS, C. Internal marketing-theory and practice. Services Marketing in a Changing Environment. Chicago: American Marketing Association, 1981. p. 41-7.

GUMMESSON, E. Lip service-a neglected area in services marketing. Journal of Services Marketing, v. 1, n. 1, p. 19-23, 1987. Disponível em: <http://dx.doi.org/10.1108/eb059585>. Acesso em: 02 de nov de 2014.

HAIR, J. F.; BABIN, B.; MONEY, A. H.; SAMOUEL, P. Fundamentos de métodos de pesquisa em administração. Porto Alegre: Bookman, 2005.

KELLER, S. B.; LYNCH, D. F.; ELLINGER, A. E.; OZMENT, J.; CALANTONE, R. The impact of internal marketing efforts in distribution service operations. Journal of Business Logistics, v. 27, n. 1, p. 109-137, 2006. Disponível em: <http://onlinelibrary.wiley.com/ doi/10.1002/j.2158-1592.2006.tb00243.x/epdf>. Acesso em: 25 de nov de 2014.

KOHLI, A. K.; JAWORSKI, B. J. Market orientation: the construct, research propositions, and managerial implications. The Journal of Marketing, v. 54, n.1, p. 1-18, 1990. Disponível em: $<$ http://www.jstor.org/stable/1251866>. Acesso em: 14 de nov de 2014.

LINGS, Ian N. Internal market orientation: Construct and consequences. Journal of Business Research, v. 57, n. 4, p. 405-413, 2004. Disponível em: <http://www.sciencedirect.com/science/ article/pii/S0148296302002746?via\%3Dihub>. Acesso em: 06 de jan de 2015.

LINGS, I. N.; GREENLEY, G. E. Measuring internal market orientation. Journal of Service Research, v. 7, n. 3, p. 290-305, 2005. Disponível em: <http://jsr.sagepub.com/content/7/3/290. full.pdf+html>. Acesso em: 02 de nov de 2014.

. Internal market orientation and market oriented behaviors. Journal of Service Management, v. 21, n. 3, p. 321-343, 2010. Disponível em: <doi:10.1016/S0148-2963(02)00274-6>. Acesso em: 10 de jan de 2015.

MAINARDES, E. W.; CERQUEIRA, A. S. Measuring the internal-market orientation in the public sector. Ekonomski Vjesnik/Econviews: Review of contemporary business, entrepreneurship and economic issues, v. 28, n. S, p. 53-70, 2015. Disponível em: <http://hrcak.srce. hr/ojs/index.php/ekonomski-vjesnik/article/view/3093/1840>. Acesso em: 20 de jun de 2015. 
MALHOTRA, N. K. Pesquisa de marketing: uma orientação aplicada. Porto Alegre: Bookman Editora, 2012.

MATTAR, F. N. Pesquisa de Marketing. São Paulo: Editora Atlas, 1996.

PACHECO, R. S. Política de recursos humanos para a reforma gerencial: realizações do período 1995-2002. Revista do Serviço Público, v. 53, n. 4, p. 79-106, 2014. Disponível em: $<$ http://seer.enap.gov.br/index.php/RSP/article/view/295/301>. Acesso em: 15 de fev de 2015.

POLLITT, C.; BOUCKAERT, G. Public Management Reform: A comparative analysis-new public management, governance, and the Neo-Weberian state. Oxford University Press, 2011.

RAFIQ, M.; AHMED, P. K. The scope of internal marketing: defining the boundary between marketing and human resource management. Journal of Marketing Management, v. 9, n. 3, p. 219-232, 1993. Disponível em: <http://www.tandfonline.com/doi/pdf/10.1080/026725 7X.1993.9964234\#.Ve8XkRFViko>. Acesso em: 03 de fev de 2015.

SASSER, W. E.; ARBEIT, S. P. Selling jobs in the service sector. Business Horizons, v. 19, n. 3, p. 61-65, 1976. Disponível em: <http://www.sciencedirect.com/science/article/ pii/0007681376900537>. Acesso em: 02 de nov de 2014.

SOLIMAN, E. M. The Relationship Between Internal Marketing Orientation and Employee Job Satisfaction in Public Sector. International Journal of Learning and Development, v. 3, n. 5, p. 111-120, 2013. Disponível em: <http://www.macrothink.org/journal/index.php/ijld/ article/view/4485/3727>. Acesso em: 15 de fev de 2015.

WALKER, R. M.; BREWER, G. A.; BOYNE, G. A.; AVELLANEDA, C. N. Market orientation and public service performance: new public management gone mad? Public Administration Review, v. 71, n. 5, p. 707-717, 2011. Disponível em: <http://onlinelibrary.wiley.com/ doi/10.1111/j.1540-6210.2011.02410.x/pdf>. Acesso em: 20 de jun de 2015.

ZAMPETAKIS, L. A.; MOUSTAKIS, V. Fostering corporate entrepreneurship through internal marketing: Implications for change in the public sector. European Journal of Innovation Management, v. 10, n. 4, p. 413-433, 2007. Disponível em: <http://dx.doi. org/10.1108/14601060710828754>. Acesso em: 12 de jan de 2015.

Recebido em: 5-2-2017

Aprovado em: 26-04-2017

Avaliado pelo sistema double blind review.

Editor: Elmo Tambosi Filho

Disponível em http://mjs.metodista.br/index.php/roc 